Chirurg 2019 · 90 (Suppl 2):S65 https://doi.org/10.1007/s00104-019-0898-0 Online publiziert: 13. Februar 2019 (c) Springer Medizin Verlag $\mathrm{GmbH}$, ein Teil von Springer Nature 2019

\section{Originalpublikation}

Yang J, Yang JY, Yan LN et al (2018) Hepatic resection vs. transarterial chemoembolization for hepatocellular carcinoma beyond the Milan criteria with portal hypertension. Dig Liver Dis 50(7):713-719

Hintergrund. Ziel der Studie war es, die Bedeutung von Leberteilresektionen im Vergleich mit der transarteriellen Chemoembolisation (TACE) bei Patienten mit hepatozellulären Karzinomen jenseits der Milan-Kritierien mit klinisch relevantem portalem Hypertonus $\mathrm{zu}$ evaluieren.

Gemäß aktueller Richtlinien werden besagte Patienten primär lokoregionären Maßnahmen zugeführt, da ein portaler Hypertonus immer noch als relative Kontraindikation für eine Leberteilresektion angesehen wird.

Methodik. Es wurde retrospektiv eine Gesamtanzahl von 363 Patienten in die Studie eingeschlossen, welche entweder einer Leberteilresektion $(n=102)$ oder einer TACE $(n=261)$ zugeführt worden waren. Diese erfüllten jeweils die Kriterien eines klinisch relevanten portalen Hypertonus und eines die Mailand-Kriterien überschreitenden hepatozellulären Karzinoms (ein Tumorknoten $>5 \mathrm{~cm}$ oder insgesamt $\leq 3$ Tumorknoten mit einem Durchmesser von $>3 \mathrm{~cm}$ bzw. einem multifokalen Befall).

Dieser Beitrag wurde erstpubliziert in Der Chirurg (2018) 89:640-640. https://doi.org/10. 1007/s00104-018-0683-5

M. Gampe - F. Rauchfuß - U. Settmacher

Klinik für Allgemein-, Viszeral- und Gefäßchirurgie, Universitätsklinikum Jena, Jena, Deutschland

\title{
Leberteilresektion vs. trans- arterielle Chemoembolisation bei hepatozellulären Karzinomen jenseits der Mailand-Kriterien und portalem Hypertonus
}

Eine Makrogefäßinvasion wurde im Vorfeld bildmorphologisch ausgeschlossen und der Grad der portalen Hypertension entweder anhand gastroskopisch festgestellter Ösophagusvarizen oder einer mit einer Thombozytopenie vergesellschafteten Splenomegalie abgeschätzt.

Grundvoraussetzung für beide Gruppen war eine stabile Leberfunktion (maximal Child-Stadium B), fehlendem Aszites und Ausschluss einer Pfortaderthrombose. Im Falle einer TACE wurde diese in regelmäßigen Intervallen von insgesamt 1,5 bis 3 Monaten wiederholt für 2 bis 6 Zyklen.

Ergebnisse. Insgesamt zeigten sich vergleichbare Ergebnisse hinsichtlich postoperativer bzw. postinterventioneller Komplikationen sowie 30-Tages-Mortalität für beide Gruppen.

Es zeigte sich ein signifikanter Überlebensvorteil zugunsten der Leberteilresektion nach 1, 2, 3 und 5 Jahren, welcher sich auch in der Propensity Score Analyse bestätigte (Leberteilresektion [LR]: $80 \%, 63,3 \%, 48,8 \%$ und $21,7 \%$ vs. TACE: $74 \%, 56,5 \%, 26,5 \%$ und $13,6 \%$ ).

Im Vergleich der beiden Gruppenpopulationen zeigte sich bei den TACEPatienten häufiger ein bilobärer Befall. Zudem waren die Patienten signifikant älter, wobei der prozentuale Anteil der über 60-Jährigen keinen relevanten Unterschied aufwies.

Auffällig war eine höhere Mortalitätsrate von $37,5 \%$ vs. $2,5 \%(p=0,012)$ in der Gruppe der Child-B-Patienten, wel- che leberteilreseziert worden waren (insgesamt 8).

Als unabhängige prognostische Faktoren wurden in der multivariaten Analyse die Behandlungsmethode, a-Fetoprotein, Child-Klassifikation, Anzahl der Tumorknoten und das Krankheitsstadium identifiziert. Nach Matching verblieb die Anzahl der Tumorknoten als unabhängiger Risikofaktor.

Fazit. Dies Studie legt nahe, dass nach sorgfältiger Selektion einer Patientengruppe mit Nachweis eines hepatozellulären Karzinoms jenseits der MailandKriterien bei klinisch relevantem portalem Hypertonus eine Leberteilresektion ähnlich sicher einer transarteriellen Chemoembolisation erscheint mit einem deutlichen Überlebensbenefit zugunsten der Leberteilresektion. Grundvoraussetzung hierfür ist ein singulärer Tumorknoten sowie eine stabile Leberfunktion (Child A). Ob diese sehr guten asiatischen Daten direkt auf die mitteleuropäische Patientenklientel übertragen werden können, bleibt zweifelhaft. Kernpunkt ist sicher die Auswahl geeigneter Patienten.

\section{Korrespondenzadresse}

\section{Gampe}

Klinik für Allgemein-, Viszeral- und Gefäßchirurgie, Universitätsklinikum Jena Am Klinikum 1, 07747 Jena, Deutschland Maria.Gampe@med.uni-jena.de

Interessenkonflikt. M. Gampe, F. Rauchfuß und U. Settmacher geben an, dass kein Interessenkonflikt besteht. 